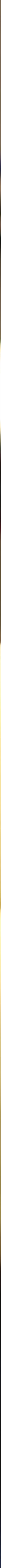




\section{Diagnosis of tuberculosis: principles and practice of using interferon- $\gamma$ release assays (IGRAs)}

\section{Educational aims}

I To provide an overview of T-cell interferon- $\gamma$ release assays (IGRAs) used for detection of tuberculosis (TB) infection.

1 To outline how these tests can be used in clinical practice for the assessment of latent TB infection (LTBI) and active TB.

\section{Summary}

One in three people worldwide are infected with Mycobacterium tuberculosis; the vast majority have latent infection. Tracking this latent reservoir is the cornerstone of TB control and prevention in the developed world [1]. But standard tools for diagnosis and prevention are more than 100 years old and inadequate to control the global TB epidemic [2]. However, the last decade has witnessed a major advance in the diagnosis of $M$. tuberculosis infection [3-5]. T-cell IGRAs are the first practical output of a decade of reinvigorated basic science research into TB and are now revolutionising diagnosis and screening of LTBI.

\section{The tuberculin skin test}

The pathway from $M$. tuberculosis exposure to disease is a multistep process that partly depends on the body's own immune defences. Tubercle bacilli may cause disease immediately or lay dormant for decades. Only $5-10 \%$ of infected individuals go on to develop active disease and the immune mechanisms underlying these disparate outcomes are poorly understood.

Diagnosis of LTBI has hitherto been defined as a positive tuberculin skin test (TST) in an asymptomatic person exposed to TB with no clinical/radiographic signs of active disease. LTBI induces a strong cell-mediated immune response, a fact exploited by the TST, which measures the delayed-type hypersensitivity response to intradermal inoculation of tuberculin purified protein derivative (PPD), a crude mixture of $>200 M$. tuberculosis proteins.

Diagnosis and treatment of LTBI is a cornerstone of TB control in low prevalence countries and targets those groups who are at increased risk of progression to TB disease by virtue of weakened or immature cellular immunity, as well as recent $\mathrm{TB}$ contacts.

The TST has several drawbacks, primarily its poor specificity in bacille Calmette-Guérin (BCG) vaccinated individuals, due to crossreactivity of PPD antigens between BCG and M. tuberculosis. The TST has poor sensitivity in
A. Lalvani

M. Thillai

Tuberculosis Research Unit, Imperial College London, UK

\section{Correspondence}

A. Lalvani

Tuberculosis Research Unit,

Department of Respiratory

Medicine, National Heart and Lung

Institute, Imperial College London,

St Mary's Campus, Norfolk Place,

London, W2 1NY,

UK

Fax: 442072628913

E-mail:a.lalvani@imperial.ac.uk

\section{Provenance}

Commissioned article,

peer reviewed

\section{Competing interests}

A. Lalvani is the inventor of several patents underpinning T-cell-based diagnosis. The Lalvani ESAT-6/ CFP-10 IFN- $\gamma$ ELISpot was commercialised by an Oxford University spin-out company (T-SPOT.TB ${ }^{\circledR}$, Oxford Immunotec Ltd, Abingdon, UK) in which Oxford University and A. Lalvani have a minority share of equity.

\section{Funding}

A. Lalvani is a Welcome Trust Senior Clinical Research Fellow and M. Thillai is a Welcome Trust Research Training Fellow

HERMES syllabus link: modules B.4, D.2.1, D.2.5

Main Image @lpkoe/istockphoto 
patient groups with the highest risk of progression to $T B$, who are specifically targeted for diagnosis and preventative treatment of latent $\mathrm{TB}$, i.e. those with suppressed or immature cellular immunity, e.g. those with HIV infection, iatrogenic immunosuppression, or young children. Finally, there are logistical difficulties in that it requires a trained health professional to perform the test and a repeat consultation for reading of the result.

The very low bacterial burden in LTBI makes it impossible to directly detect $M$. tuberculosis and the weak humoral response makes serological testing unreliable. However, M. tuberculosis infection evokes a strong T-cell response dominated by T-helper cell type 1 (Th1) CD4 T-cells which secrete interferon (IFN) $\gamma$.

\section{IGRA}

Advances in mycobacterial genomics in the 1990s resulted in the identification of a genetic segment (region of difference (RD)1) that is deleted from all strains of BCG and most environmental mycobacteria [6]. Of the nine RD1 open reading frames, Rv3875 (early secretory antigenic target (ESAT)-6) and Rv3874 (culture filtrate protein (CFP)-10) were the first to be studied and are strong targets of Th1 cells in $M$. tuberculosis infection.

We hypothesised that a T-cell response to these antigens could serve as a highly specific and sensitive marker of infection which would bypass the problem of false-positive TST results in individuals with BCG vaccination.

The rapid ex vivo enzyme-linked immunospot (ELISpot) assay developed by Lalvani enumerates individual antigen-specific T-cells that secrete IFN- $\gamma$, which appear as dark spots, where each spot is the 'footprint' of an individual T-cell specific for M. tuberculosis [4]. Spots are counted using a magnifying lens or automated reader.

The whole-blood IFN- $\gamma$ ELISA, modified from an assay originally developed for diagnosing bovine TB in cattle, measures the concentration of IFN- $\gamma$ in the supernatant of a diluted wholeblood sample after incubation with the same antigens for $24 \mathrm{~h}$. The ELISA initially used PPD, which was then replaced with ESAT-6 and CFP-10 in the ELISpot [7-9]. A third antigen (TB7.7 from RD11) has been recently incorporated and the format simplified with antigens coated onto the inner aspect of blood collection tubes enabling T-cell stimulation to happen within these tubes.

Both assays exploit the fact that T-cells from people infected with $M$. tuberculosis become
Figure 1

M. tuberculosis-infected macrophages in tuberculous granulomas induce myriad T-cell populations including $\mathrm{CD} 4+$ T-cells (the most dominant of the induced T-cells) which produce IFN- $\gamma$, but also CD8 T-cells, regulatory T-cells, CD1restricted T-cells, natural killer cells and $\gamma / \delta T$-cells.

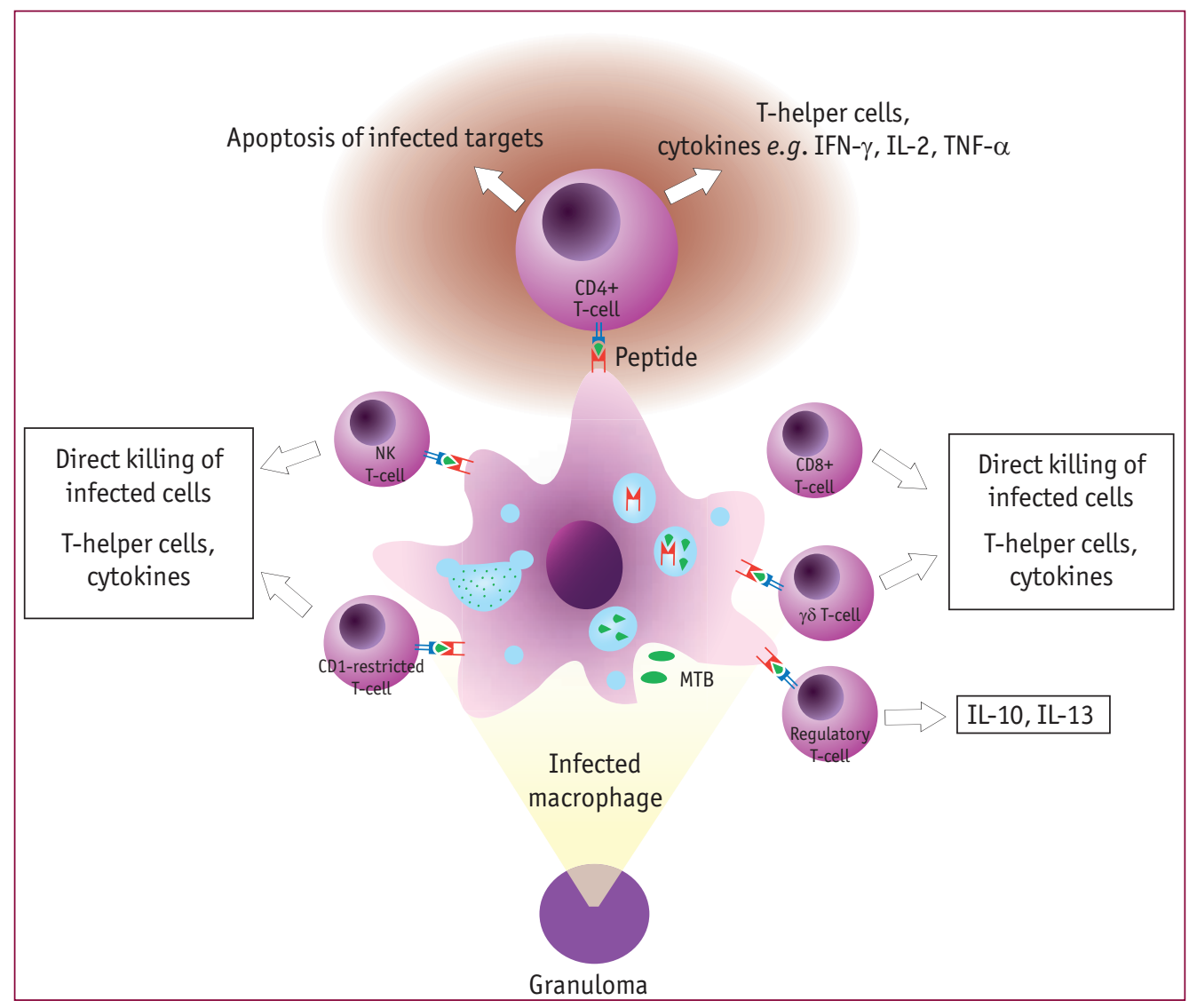


sensitised to ESAT-6 or CFP-10 in vivo and release IFN- $\gamma$ when they reencounter the same antigens ex vivo.

Both assays are commercially available as quality-controlled, regulatory-approved diagnostic test kits. The ELISpot is available as T-SPOT.TB (Oxford Immunotec Ltd, Abingdon, UK) and the ELISA as Quantiferon-TB Gold In-tube (Cellestis Inc, Carnegie, Australia). Both IGRAs have similarities (e.g. both need to be processed within $8 \mathrm{~h}$ of taking a blood sample), as well as differences (e.g. ELISpot requires separation of white blood cells, which ensures a fixed number in the assay but is technically more complex to process).

\section{From bench to bedside: validation of the scientific hypothesis}

The high diagnostic sensitivity and specificity of IGRAs was initially tested in patients with cultureconfirmed active TB and early studies established that the assays are not confounded by prior BCGvaccination or by non-TB illnesses that clinically mimic $T B$, including diseases involving immune activation. However, the lack of gold standard reference testing for LTBI makes it impossible to

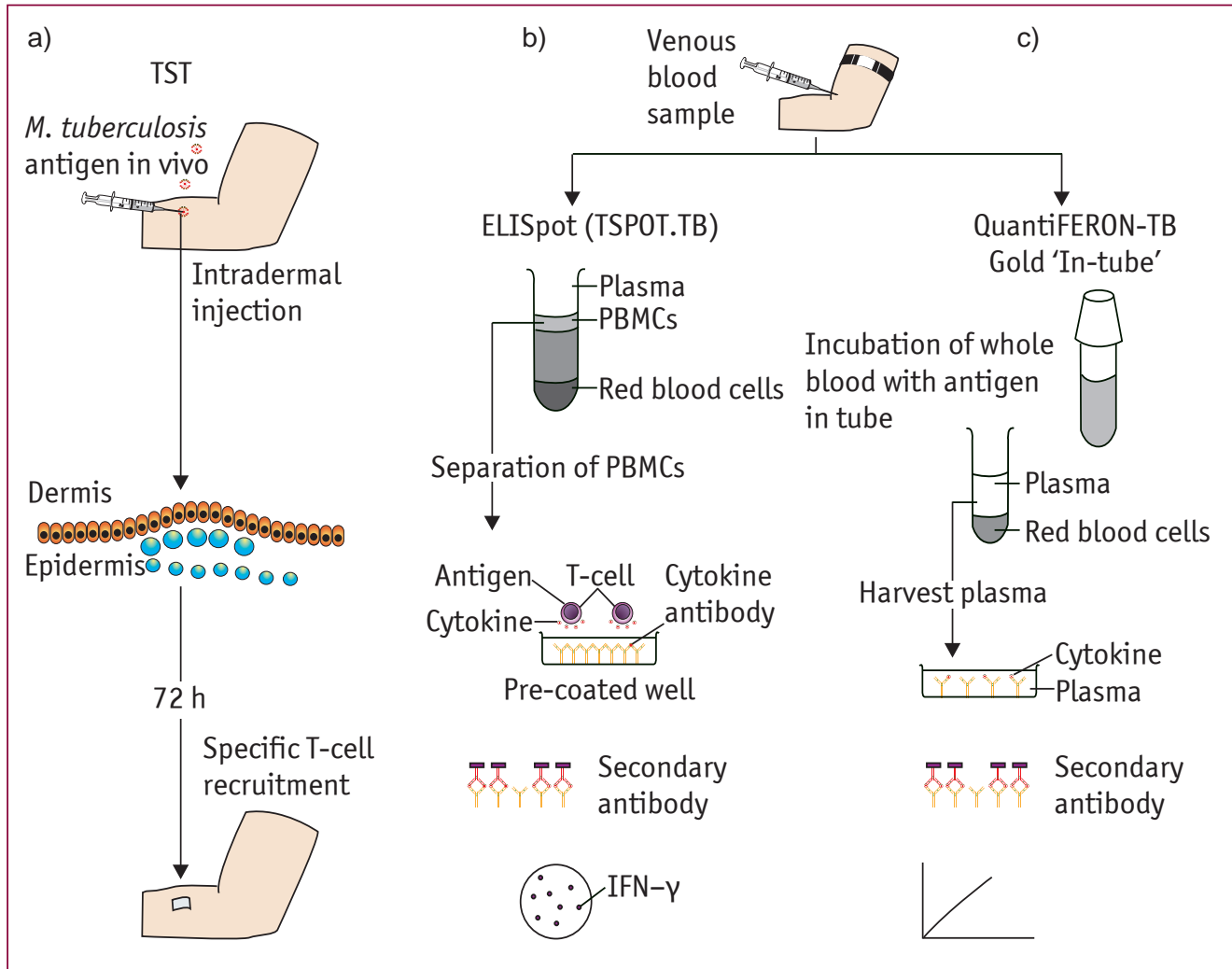

Figure 2

a) TST (purified protein derivative) is injected intradermally and the delayed-type hypersensitivity response is measured at 72 h. b) ELISpot uses mononuclear cells (including T-cells); separated from blood by density centrifugation and washed, counted, and incubated with ESAT-6 and CFP-10 in a 96-well microtitre plate for 16-20 h. In M. tuberculosis infection, T-cells recognise these antigens and secrete IFN- $\gamma$ which is captured in the immediate vicinity by antibodies specific for IFN- $\gamma$ coated on the bottom of each well. The cytokine-bound antibodies are detected with another antibody conjugated to an enzyme that catalyses a colorimetric reaction, which results in visible spots, where each spot represents the footprint of one T-cell that responded to the antigens. Spots are counted, and the frequency of $\mathrm{M}$. tuberculosisspecific T-cells is quantified. c) ELISA uses whole blood incubated with ESAT-6, CFP-10 and TB7.7 in a tube for 16-24 $h$. In M. tuberculosis infection, T-cells recognise these antigens and secrete IFN- $\gamma$. The tube is centrifuged and the plasma transferred to a 96-well microtitre plate. IFN- $\gamma$ in the plasma is captured by antibodies specific for IFN- $\gamma$ coated on the bottom of each well. The cytokine-bound antibodies subsequently are detected by another antibody conjugated to an enzyme that catalyses a colorimetric reaction. The optical density of each well is measured, and the concentration of IFN- $\gamma$ is determined using a standard curve. 
directly to quantify the diagnostic sensitivity and specificity of IGRAs in latent infection.

However, airborne transmission of M. tuberculosis is promoted by duration and proximity of contact with an infectious case, and the key determinant of infection is the amount of time spent sharing room air with an index case. We therefore postulated that if IGRAs are more sensitive and specific than TST, they should correlate more closely with the level of exposure to $M$. tuberculosis and should be independent of BCC vaccination status.

This postulate was originally tested and confirmed for ELISpot in a series of contact-tracing investigations and institutional outbreaks in 2001-2005 [10-12], notably in a UK school outbreak in which TB exposure of 535 students was precisely quantified based on the number of classes students shared with the source case. ELISpot correlated significantly more closely with exposure to $M$. tuberculosis than TST on these measures of exposure. Further outbreak investigations with both IGRA platforms around the world subsequently confirmed these findings [13-15]. These and other studies have confirmed the very high specificity of both assays, which is close to $100 \%$.

ELISpot performs well in patients co-infected with HIV (published data in this population is less extensive with ELISA) and, unlike the TST, it is robust in individuals with low CD4 counts [16]. In young children, ELISpot seems to have higher sensitivity than the TST, while ELISA may have similar sensitivity [17]. In this population, ELISpot results appear to be unaffected by HIV infection, malnutrition and age under 3 years [18]; however, published studies in children provide conflicting results on test performance and there is as yet no clear consensus $[19,20]$.

The high sensitivity of IGRAs in young children and immunosuppressed populations (e.g. iatrogenically suppressed patients with rheumatic disorders before initiating anti-tumour necrosis factor therapy [21]) allows more accurate targeting of latently infected people at the highest risk of progression. Indeterminate results are more common with ELISA and are associated with immunosuppression, young age and old age, whereas indeterminate results with ELISpot are rare in all risk groups [22].

While cross-sectional studies are a key evidence base supporting the notion that positive IGRA results reflect $L T B I$, they do not indicate whether TB contacts with positive IGRA results are at risk of progression to TB disease. Without longitudinal clinical outcomes, we cannot know

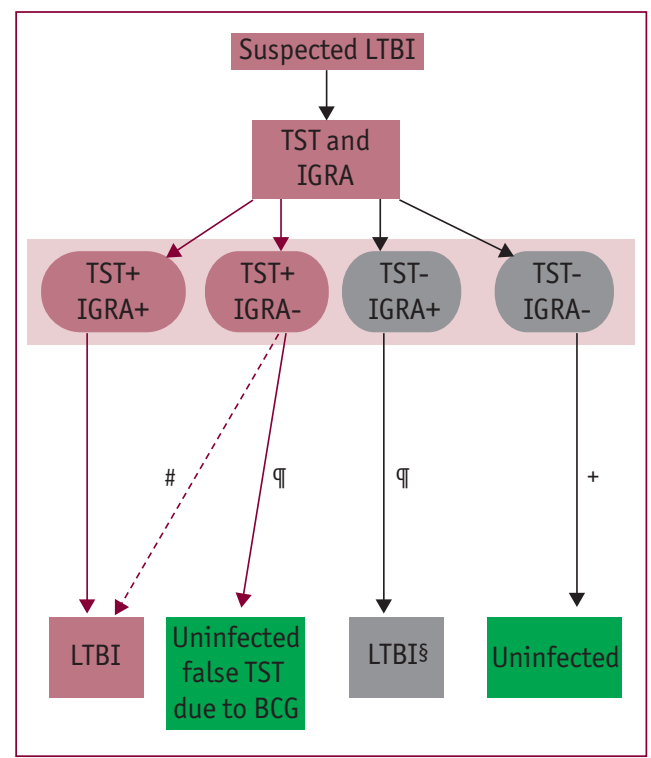

Figure 3

Negative TST and IGRA indicates no infection. Positive TST and IGRA indicates LTBI. \#: With strong evidence for LTBI (e.g. >25 mm TST, ulcerating Mantoux, calcified lesions on chest radiographs), the possibility of false-negative IGRA must be considered. "I: A positive TST/IGRA must be evaluated carefully in immunocompromised patients in whom any positive TB test is significant. ${ }^{+}$: Dual negative TST and IGRA results normally indicate the exclusion of TB infection but negative results should be evaluated carefully in immunocompromised patients. ${ }^{\text {: }}$ : Risk of active TB development in this group is not yet known. Longitudinal contact studies have defined a group of TST-negative individuals with transiently positive IGRAs, raising the possibility that some TST-negative contacts acquire and spontaneously clear transient M. tuberculosis infection. Red arrow: NICE guidance.

whether IGRA-positive contacts actually stand to benefit from preventive treatment.

Recently, key studies correlating baseline IGRA results with clinical outcomes over 2 years' follow-up have been published for both IGRA formats, and each indicates that positive IGRA results in recent contacts have prognostic value for subsequent development of TB. These studies included children [23] and HIV-infected [24] subjects as well as immunocompetent adults [25].

The confirmation of the greater prognostic power of IGRAs compared with the TST will require further larger longitudinal studies but it is already clear that when using a lower threshold of $5 \mathrm{~mm}$ to determine a positive TST response, the resulting lower TST specificity means that significantly more contacts would need to be given chemoprophylaxis on the basis of positive TST results than on the basis of a positive IGRA to prevent a similar number of incident TB cases. 


\section{Clinical utility of IGRAs in active TB}

IGRAs are tests of infection, rather than disease, and cannot therefore distinguish between latent infection and active TB. However, since infection with $M$. tuberculosis is a prerequisite for active $T B$, a negative test result might in theory rapidly exclude TB from the differential diagnosis. Multiple studies of the accuracy of IGRAs in active TB have used culture-confirmed disease or clinically highly probable cases (e.g. those that are culture negative but respond to anti-TB therapy) as a gold standard.

Both IGRAs have been shown to have higher sensitivity than the TST, while ELISpot is signifcantly more sensitive than ELISA [26]. However, neither of the currently commercially available IGRAs have a high enough sensitivity to be used as a stand-alone rule-out test [27].

The largest study of the potential role of IGRAs in active TB compared the TST, ELISpot and the next-generation ELISpot ${ }^{\mathrm{PUS}}$ assay (incorporating a third RD1-encoded antigen, Rv3879c) [28]. Diagnostic sensitivity was significantly higher for ELISpot ${ }^{\text {PUS }}$ than for ELISpot and, when used with the TST, ELISpot ${ }^{\text {PLS }}$ gave a combined diagnostic sensitivity of $99 \%$, so dual negative results enabled rapid exclusion of active TB even in patients with moderate-to-high pre-test probability of disease.

Conversely, a dual positive TST and IGRA can help guide decisions about immediate management in patients with severe disease prior to culture confirmation and in patients with extrapulmonary disease, for whom culture is frequently negative. When used to support a diagnosis of active TB, both the TST and IGRA results should be interpreted as part of the overall clinical context.

In situ analysis of disease with ELISpot is sensitive and specific in pleural fluid in tuberculous pleuritis [29,30], bronchoalveolar lavage in smear-negative pulmonary TB [31], and cerebrospinal fluid in TB meningitis [32]. However, the evidence-base is preliminary and use of IGRAs in site of disease samples lacks regulatory approval.

\section{Incorporation of IGRAs as pivotal tools in TB control policy}

Accurate diagnosis of LTBI in contacts from an infectious source will lessen the number of people inappropriately treated on the basis of false-positive TST results caused by prior BCG

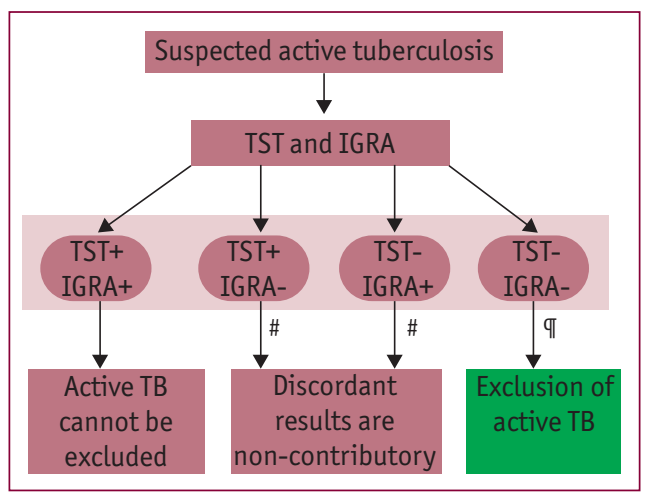

vaccination and IGRAs are now integrated into many national guidelines for the diagnosis of LTBI but recommendations differ between continents. In Europe, most countries have followed the recommendations of the UK National Institute for Health and Clinical Excellence in using IGRAs to confirm LTBI in those with a positive TST and in individuals in whom the TST is considered unreliable (e.g. those with suppressed cellular immunity [33]), thereby enabling more reliable detection of LTBI in immunosuppressed patients.

IGRAs are more expensive than the TST, due to the cost of test kits and the need for specialist laboratory processing, but health-economic analysis has consistently shown that they become cost effective for the diagnosis and treatment of LTBI when incorporated into programmes to control $\mathrm{TB}$, by reducing the costs associated with unnecessary chemoprophylaxis and morbidity in contacts with false-positive TST results [34, 35].

\section{Recent developments and future directions}

In addition to further immunogenic but highly specific $M$. tuberculosis antigens (such as Rv3879c in the ElISpot ${ }^{\text {PUS }}$ and Rv2645 in the latest version of the in-tube ELISA (QuantiferonTB Gold In-tube), measurement of additional downstream chemokines and cytokines induced by IFN- $\gamma$ (e.g. IFN- $\gamma$ inducible protein-10) increases diagnostic sensitivity while maintaining high specificity [36].

CD4+ effector T-cells measured by the assays are in dynamic equilibrium with $M$. tuberculosis antigen load in vivo but, while the ELISpot response has been shown to decrease in general with treatment of active $T B$, the rate of decline is highly variable and only a minority are negative by the end of treatment. The result of an IGRA cannot, therefore, be accurately interpreted in patients who have previously been treated for active TB or LTBI.

\section{Figure 4}

Positive TST and IGRA results may help with early initiation of presumptive treatment prior to culture confirmation and in those with extrapulmonary disease, for whom culture is frequently negative. Discordant results do not contribute to the diagnostic work up. Negative TST/IGRA results can be used as a 'rule-out test' to reliably exclude active $T B$ with high negative predictive value. \#: A positive TST/ IGRA must be evaluated carefully in immunocompromised patients in whom any positive TB test is significant. ": Dual negative TST/IGRA results normally indicate the exclusion of active TB but negative results should be evaluated carefully in immunocompromised patients. 
Although current IGRAs cannot be used to monitor treatment of LTBI and active TB disease, incorporation of additional cytokines raises the possibility of monitoring treatment response and cure [37]. Simultaneous measurement of secretion of interleukin (IL)-2 and IFN- $\gamma$ by $M$. tuberculosis-specific T-cells reveals T-cell cytokine profiles (defined as the relative proportions of antigen-specific T-cells secreting IFN- $\gamma$ only, IFN- $\gamma$ and IL-2 or IL-2 only) that correlate with bacterial and antigen load as well as with treatment response [38].

The current generation of commercially available IGRAs, while a 100-year update of the TST, is in reality an evolving work in progress that builds on our state of knowledge from the 1990s. The next generation of T-cell assays will incorporate more antigens and measure multiple cytokines and they promise to provide enhanced diagnostic sensitivity and more useful information about in vivo bacterial burden and clinical status than existing assays based only on IFN- $\gamma$.

\section{References}

1. World Health Organization. Global tuberculosis control - epidemiology, strategy, financing. Geneva, World Health Organisation, 2009. Available from: www.who.int/tb/publications/global_report/2009/pdf/full_report.pdf

2. World Health Organization. The Global Plan to stop TB. Geneva, World Health Organization, 2006. Available from: http://whqlibdoc.who.int/hq/2006/WHO_HTM_STB_2006.368_eng.pdf

3. Lalvani A, Pathan AA, McShane H, et al. Rapid detection of Mycobacterium tuberculosis infection by enumeration of antigen-specific T cells. Am J Respir Crit Care Med 2001; 163: 824-828.

4. Lalvani A, Pathan AA, Durkan H, et al. Enhanced contact tracing and spatial tracking of Mycobacterium tuberculosis infection by enumeration of antigen-specific T cells. Lancet 2001; 357: 2017-2021.

5. Barnes PF. Diagnosing latent tuberculosis infection. The 100-year upgrade. Am J Respir Crit Care Med 2001; 163: 807-808.

6. Behr MA, Wilson MA, Gill WP, et al. Comparative genomics of BCG vaccines by whole-genome DNA microarray. Science 1999; 284: 1520.

7. Mori T, Sakatani M, Yamagishi F, et al. Specific detection of tuberculosis infection: an interferon- $\gamma$-based assay using new antigens. Am J Respir Crit Care Med 2004; 170: 59-64.

8. Brock I, Weldingh K, Lillebaek T, Follmann F, Andersen P. Comparison of tuberculin skin test and new specific blood test in tuberculosis contacts. Am J Respir Crit Care Med 2004; 170: 65-69.

9. Barnes PF. Diagnosing latent tuberculosis infection: turning glitter to gold. Am J Respir Crit Care Med 2004; 170: 5-6.

10. Ewer K, Deeks JJ, Alvarez L, et al. Comparison of T-cell-based assay with tuberculin skin test for diagnosis of Mycobacterium tuberculosis infection in a school tuberculosis outbreak. Lancet 2003; 361: 1168-1173.

11. Richeldi L, Ewer K, Losi M, et al. T cell-based tracking of multidrug resistant tuberculosis infection after brief exposure. Am J Respir Crit Care Med 2004; 170: 288-295.

12. Shams H, Weis SE, Klucar P, et al. Enzyme-linked Immunospot and tuberculin skin testing to detect latent tuberculosis infection. Am J Respir Crit Care Med 2005; 172: 1161-1168.

13. Diel R, Nienhaus A, Lange C, Meywald-Walter K, Forssbohm M, Schaberg T. Tuberculosis contact investigation with a new, specific blood test in a low-incidence population containing a high proportion of BCG-vaccinated persons. Respir Res 2006 17; 7: 77.

14. Nakaoka H, Lawson L, Squire SB, et al. Risk of tuberculosis among children. Emerg Infect Dis 2006; 12: 1383-1388.

15. Zellweger JP, Zellweger A, Ansermet S, de Senarclens B, Wrighton-Smith P. Contact tracing using a new T-cell-based test: better correlation with tuberculosis exposure than the tuberculin skin test. Int J Tuberc Lung Dis 2005; 9: 1242-1247.

16. Dheda K, Lalvani A, Miller RF, et al. Performance of a T-cell-based diagnostic test for tuberculosis infection in HIVinfected individuals is independent of CD4 cell count. AIDS 2005; 19: 2038-2041.

17. Lalvani A, Millington KA. T cell-based diagnosis of childhood tuberculosis infection. Curr Opin Infect Dis 2007; 20: 264-271.

18. Liebeschuetz S, Bamber S, Ewer K, Deeks J, Pathan AA, Lalvani A. Diagnosis of tuberculosis in South African children with a T-cell-based assay: a prospective cohort study. Lancet 2004; 364: 2196-2203.

19. Kampmann B, Whittaker $E$, Williams A, et al. Interferon-gamma release assays do not identify more children with active TB than TST. Eur Respir J 2009; 33: 1371-1379.

20. Nicol MP, Davies MA, Wood K, et al. Comparison of T-SPOT.TB assay and tuberculin skin test for the evaluation of young children at high risk for tuberculosis in a community setting. Pediatrics 2009; 123: 38-43.

21. Lalvani A, Millington KA. Screening for tuberculosis infection prior to initiation of anti-TNF therapy. Autoimmun Rev 2008; 8: 147-152.

22. Menzies D, Pai M, Comstock G. Meta-analysis: new tests for the diagnosis of latent tuberculosis infection: areas of uncertainty and recommendations for research. Ann Intern Med 2007; 146: 340-354.

23. Bakir M, Millington KA, Soysal A, et al. Prognostic value of a T-cell-based, interferon- $\gamma$ biomarker in children with tuberculosis contact. Ann Intern Med 2008; 149: 777-787.

24. Aichelburg MC, Rieger A, Breitenecker F, et al. Detection and prediction of active tuberculosis disease by a wholeblood interferon- $\gamma$ release assay in HIV-1-infected individuals. Clin Infect Dis 2009; 48: 954-962.

25. Diel R, Loddenkemper R, Meywald-Walter K, Niemann S, Nienhaus A. Predictive value of a whole blood IFN- $\gamma$ assay for the development of active tuberculosis disease after recent infection with Mycobacterium tuberculosis. Am J Respir Crit Care Med 2008; 177: 1164-1170.

26. Lalvani A. Diagnosing tuberculosis infection in the 21st century: new tools to tackle an old enemy. Chest 2007; 131: 1898-1906.

27. Goletti D, Stefania C, Butera 0, et al. Accuracy of immunodiagnostic tests for active tuberculosis using single and combined results: a multicenter TBNET-Study. PLoS ONE. 2008; 3: e3417.

28. Dosanjh DP, Hinks TS, Innes JA, et al. Improved diagnostic evaluation of suspected tuberculosis. Ann Intern Med 2008; 148: 325-336. 
29. Wilkinson KA, Wilkinson RJ, Pathan A, et al. Ex vivo characterization of early secretory antigenic target 6-specific T cells at sites of active disease in pleural tuberculosis. Clin Infect Dis 2005; 40: 184-187.

30. Jafari C, Ernst M, Strassburg A, et al. Local immunodiagnosis of pulmonary tuberculosis by enzyme-linked immunospot. Eur Respir J 2008; 31: 261-265.

31. Jafari C, Ernst M, Kalsdorf B, et al . Rapid diagnosis of smear-negative tuberculosis by bronchoalveolar lavage enzyme-linked immunospot. Am J Respir Crit Care Med 2006; 174: 1048-1054.

32. Thomas MM, Hinks TS, Raghuraman S, et al. Rapid diagnosis of Mycobacterium tuberculosis meningitis by enumeration of cerebrospinal fluid antigen-specific T-cells. Int J Tuberc Lung Dis 2008; 12: 651-657.

33. National Collaborating Centre for Chronic Conditions. Tuberculosis: clinical diagnosis and management of tuberculosis, and measures for its prevention and control. London, Royal College of Physicians, 2006. Available from: www. rcplondon.ac.uk/pubs/books/TB/Tuberculosis2.pdf

34. Diel R, Nienhaus A, Lange C, Schaberg T. Cost-optimisation of screening for latent tuberculosis in close contacts. Eur Respir J 2006; 28: 35-44.

35. Wrighton-Smith P, Zellweger JP. Direct costs of three models for the screening of latent tuberculosis infection. Eur Respir J 2006; 28: 45-50.

36. Ruhwald M, Bodmer T, Maier C, et al. Evaluating the potential of IP-10 and MCP-2 as biomarkers for the diagnosis of tuberculosis. Eur Respir J 2008; 32: 1607-1615.

37. Liu XQ, Dosanjh D, Varia H, et al. Evaluation of T-cell responses to novel RD1- and RD2-encoded Mycobacterium tuberculosis gene products for specific detection of human tuberculosis infection. Infect Immun 2004; 72: 2574-2581.

38. Millington KA, Innes JA, Hackforth S, et al. Dynamic relationship between IFN-gamma and IL-2 profile of Mycobacterium tuberculosis-specific T cells and antigen load. J Immunol 2007; 178: 5217-5226.

\section{Further reading}

Lalvani A. Diagnosing tuberculosis infection in the 21st century: new tools to tackle an old enemy. Chest 2007;

131: 1898-1906.

Pai M, Zwerling A, Menzies D. Systematic review: T-cell-based assays for the diagnosis of latent tuberculosis infection: an update. Ann Intern Med 2008; 149: 177-184.

Lalvani A, Millington KA. T-cell interferon- $\gamma$ release assays: can we do better? Eur Respir J 2008; 32: 1428-1430. 